\title{
Cooperative and Opportunistic Transmission in Wireless and Mobile Ad-Hoc Networks
}

\author{
Suyog S. Nyati and Suresh V. Limkar
}

\begin{abstract}
G technology is getting more popular in wireless and mobile network domain. Opportunistic and cooperative transmission techniques are important aspects for communication in wireless and mobile networks. But these techniques are facing routing and forwarding issues as well as co-channel interference and lack of central controller problems. For these problems various techniques to routing and forwarding data packets are proposed in recent years. In order to comprehensive understanding of these techniques considering cooperative and opportunistic transmission, we did brief survey of useful techniques involved in the cooperative and opportunistic network in this paper.
\end{abstract}

Index Terms-Mobile ad-hoc network (MANET), wireless network, survey.

\section{INTRODUCTION}

Wireless networks as well as mobile network has recently attracted growing interest, and has been used as key technology for the next generation. As the better mobility is achieve by using these technology the popularity of these techniques is increasing. The devices are smaller and cheaper to use this technique that's why these networks are being more convenient to use in regular life. To give better Quality of Service (QoS) we have to give the best and efficient way to give the reliable and efficient connectivity over the network [1]. In opportunistic networks, mobile nodes are enabled to communicate with each other even if a route connecting them never exists. Furthermore, nodes are not supposed to possess or acquire any knowledge about the network topology, which (instead) is necessary in traditional MANET routing protocols. Routes are built dynamically, while messages are en route between the sender and the destination(s), and any possible node can opportunistically be used as next hop, provided it is likely to bring the message closer to the final destination as next hop [2].

As the nodes which sense the wireless signals are battery powered, energy efficiency becomes one of the key challenges in wireless network [1], [3]. Due to mobility in the wireless ad-hoc networks, links are not permanent or constant. We cannot achieve ene-to-end transmission in wireless networks due to time varying and transient links. To communicate over this type of networks, opportunistic networks helps to enable user communication in

Manuscript received April 7, 2013; revised May 22, 2013

S. S. Nyati is with the Department of Computer Engineering, Pune Institute of Computer Technology, Pune. Maharashtra, India 411043 (e-mail: suyognyatirsp@gmail.com).

S. V. Limkar is with the Computer Engineering Department of AISSMS's IOIT, Pune, Maharashtra, India (e-mail: sureshlimkar@gmail.com). environments where disconnection and reconnection are likely and link performance is extremely non stationary [4]. But this solution suffers the Quality of Service (QoS) issue. To achieve QoS there are some routing algorithm like Opportunistic packet scheduling and auto rate (OSAR) [5], Automatic repeat request (ARQ) based relaying scheme [6] etc. which rout the path from sender to destination to achieve possibly better QoS and give reliability of connection to user.

The rest of the paper is organized as follows.

In the section II we discussed basic concepts. In Section III the Problems addressed in Wireless and Mobile Ad-Hoc networks summarize. In Section IV Routing and forwarding techniques are discussed, in Section V Observations are taken, Section VI covers Proposed Idea and finally we concluded in Section VII.

\section{BASIC CONCEPT}

\section{A. Cooperative and Opportunistic Network Architecture}

In wireless or mobile network direct end-to-end communication is difficult as nodes connect to infrastructure when they want and disconnect again this leads to transient link between nodes. So if any node wants to directly communicate with another node and if that another node is not available or disconnected at that time then there should be some techniques to receive message up to destination when that node will be connected to infrastructure.

Cooperative and opportunistic transmission overcome this drawback and help to communicate this type of lossy network. In opportunistic environment although nodes are typically mobile, there are some fixed node present as well. Here node may discover each other and can communicate by using all kind of mechanism or communicate over communication media, such as Bluetooth, WiFi, RFID, cellular based technologies etc. from these some of them act may act as point of access towards the fixed internet or satellite link.

The network is typically separated into several network partitions, which is called regions. As previously we had discussed that we cannot give assurance for existence of end-to-end path between sender and receiver furthermore link performance is typically highly variable or extreme and thus even if there is an end-to-end path between source and the destination it may last only for a brief and unpredictable period of time.

To solve this issue, node mobility and local forwarding can be exploited for data transferring: the network nodes can store and carry data around while they are moving, and then forward the data during opportunistic contacts. During these opportunistic contacts, entire chunks of a message can be 
transferred from one storage place to a storage place in another node. It follows that nodes may transfer data to the destination either through single-hop transmissions or using the multihop paradigm (i.e., along a path that is expected to reach the destination). The store-carry-forward message switching mechanism is used in the intermediate nodes between a source and a destination

\section{B. Network Scenario}

Opportunistic networks are more generic and not necessarily limited to the Internet-like architecture with gateways, as often assumed by DTN [7]. Wireless networks traditionally viewed as a source of unreliability that needs to be mitigated, recent research suggests exploiting the channel fluctuations opportunistically when and where the channel is strong [8]. At first error compensation, link status estimation and fair scheduling was not considered for wireless ad hoc networks which exploit some issues in scheduling and transmitting packets over some bad links [9]. Also error control on the source node is not useful in wireless Ad-Hoc Network [6].

The previous algorithm did not consider the interaction among neighbouring transmitter i.e. sender make its local decision to maximize its local performance. Instead of that cooperatively selecting the outgoing link remarkably increases the network throughput [10]. All previous schemes on multiuser diversity are based on wireless cellular networks where a base station acts as the central controller and control channels are available for channel state feedback. The methodology to design MAC protocol in the contention-based wireless ad hoc networks is much different from that in wireless cellular networks [5].

Traditional topology control algorithms rely on the assumption of static wireless links, so they cannot exploit the opportunity existed in time-varying wireless links in real deployments where, the opportunistic topology control which is useful to exploit the opportunity existed in time-varying wireless links in real deployments [3]. Most of the existing topology control algorithms no matter that they concern QoS or not for example they only concern about link is connected or disconnected but not the QoS given by the link [1].

\section{PRoblems AdDRESSED IN WIRELESS AND MoBILE AD-HOC NETWORKS}

In wireless and mobile Ad-Hoc networks relying on only one sink, make some issues in communication. Also sensor nodes are always battery powered as well as the nodes are mobile nodes, so energy efficiency is one of the major issues in wireless sensor nodes [3]. Besides this energy efficiency there is also some application dependent QoS requirement such as delay, throughput and packet reliability. In the connected and disconnected region, a large percentage of links reside in the transitional region with fluctuating link qualities [1]. Because of this how to improve the fragile end-to-end network services in presence of interference-prone wireless communications and volatile network topology in MANET is the main problem. Also high-mobility incurs fast route breakage, which in turn results in the failure of end-to-end data delivery [7].

Opportunistic routing class exploits both the temporal diversity and the broadcast nature of the wireless propagation. It uses broadcast communication instead of traditional unicast one to provide connectivity in presence of hostile wireless propagation condition. In this the sender send packet to all neighbors which can assure more resilience to lossy network. But because of this the all neighbor nodes are participate in this and forward the same packet where some of them are useless as they are far from receiver. So it cause in network flooding [4]. Also fading is common in wireless networks because of multiple paths between source \& destination. Without cooperation among transmitter the higher bandwidth requirement leads to sever effective throughput degradation. Here the more problem addressed which are - First, with a hidden terminal there is inequality in channel contention among nodes in wireless ad hoc networks, which can result in severe overall performance inefficiency. Second, with the shared wireless medium, co-channel interference has a deep impact on rate selection and flow scheduling in wireless ad hoc networks. Hence, neighbouring transmitters should jointly determine the "on-peak" flows and their corresponding rate in a distributed way. Third, different QoS requirements of the system correspond to different optimization targets [8]. The co-channel interference has deep impact on the link scheduling due to the shared media in wireless ad hoc networks. For this in wireless Ad-Hoc Network total channel capacity can be maximized by picking the user with the best channel to transmit. But while selecting the links with good channel conditions, it is also important to consider the fairness among the flows. To schedule a link should not be barely based on its channel quality but also the achieved throughput of its own and the neighbouring flows. Also without the help of any infrastructure node, the scheduling policy should be executed in a distributed way [10].

User applications require reliable information delivery. So on wireless network to achieve more reliable delivery wireless ad hoc network with multiple co-operative relays was used. But it has some error control issues which are different than the normal error control mechanism which are considered at the source but not at the relay nodes [6]. A wireless ad hoc network consists of a number of nodes communicating with each other on wireless links without infrastructure support. A multihop ad hoc network is an ad hoc network in which the packets of a traffic flow are relayed by one or more intermediate nodes before they reach the destination. To support different types of multimedia applications, providing various quality of service (QoS) guarantees for multihop flows is an important issue in wireless ad hoc networks [9].

The popularity of multimedia content sharing among the Internet users had promoted a new kind of networking where the content dissemination is determined by the interests of users rather than pre-specified destinations. However, such mobile nodes based content dissemination may suffer the underlying intermittent connectivity due to the inherent limitations such as short radio transmission range, sporadic node densities and constrained power [11]. For a given node pair, the link quality may be too poor to transmit any data even at the lowest data rate or may be so good that a high data rate 
can be achieved. This channel variation creates problems in the wireless ad-hoc networks [5].

\section{ROUTING AND FORWARDING TECHNIQUES TO OVERCOME PROBLEMS}

Since every coordinator always turns on its wireless radio signal we can opportunistically connect to sink through coordinator. As energy efficient opportunistic topology control problem is NP hard problem the greedy solution was proposed with a deep analysis of end to end performance requirements over opportunistic control [3]. Specifically a distributed cooperative rate adaptation (CRA) is useful scheme to achieve energy efficiency in wireless ad hoc networks. Given the neighbouring link information, each node adopts a rate selection algorithm to calculate the most energy-efficient setting of rates for all links in its maximum interference range. Then the node consults the neighbouring nodes about the feasibility of this new physical layer (PHY) rate. The procedure is repeated until it converges. Moreover, a cooperative and opportunistic scheduling (COS) scheme is beneficial to exploit multi-user diversity for wireless ad hoc networks while fulfilling given quality of service (QoS) requirements. By exchanging interference information, average channel conditions, and QoS factors among neighbouring nodes in a two-hop transmission range, cooperative scheduling aims to find out the globally optimal set of simultaneously transmitting flows. In addition, through cooperation, some transmissions are deferred to favor some other links that have not achieved their QoS requirements [8]. Expected transmission count (ETX) is also very useful in opportunistic transmission. For QoS topology control problem opportunistic transmission based ETX is useful. The centralized and distributed solutions employ opportunistic transmission to achieve better efficiency [1]. Opportunistic forwarding can be useful to solve the end to end connectivity problem where in a non-sparse high-mobility opportunistic net-work, a node potentially may have one or more connected neighboring nodes that can lead to the destination of the broken route all the time. Flooding can be functioning in such challenging scenarios while routing cannot. In fact, uncontrolled flooding is the most primitive [7].

For throughput and spectral efficiency in ad-hoc networks an analytical model for end to end ARQ based relaying scheme such as SR and IR scheme are useful, where every intermediate node combines signals receiving from all preceding nodes before retransmitting. Also impact of the channel quality and protocol parameters is evaluated and the performance conflicts between spectral efficiency and throughput rate can be analyzed [6].

For disruption tolerant network opportunistic DHT based routing (ODR) protocol in which a candidate set is selected by comparing the distance between the forwarder to receiver and its neighbor to receiver. Only that candidate set will follow the packet and rest of nodes are not included in this [4]. "Robust Opportunistic Scheduling for Ad Hoc Networks" (ROSA) is scheduling algorithm which is designed for infrastructure wireless networks which can be adapted to multihop ad hoc networks. By utilizing the link status information provided by
ROSA the adapted algorithm performs distributed scheduling opportunistically. MAC layer blocking problem and solution, Link state estimation, Medium access abuse problem and solution, Adapting BGFS-EBA to distributed environment are covered by ROSA framework [9]. A distributed scheduling algorithm, which is inspired by a greedy algorithm in graph theory to find the maximum weighted independent set, is also used for opportunistic scheduling. In this scheme, the links exchange their channel information and determine their own transmission priorities. Through such a priority-based link scheduling algorithm, a link with better channel condition would access the channel with higher probability, while the fairness requirements are taken into account [10].

To exploit the multiuser diversity in the CSMA/CA based wireless ad hoc networks a new MAC protocol is used, which is the opportunistic packet scheduling and auto rate (OSAR) protocol. This protocol takes advantage of both multiuser diversity and rate adaptation. Based on Multi-RTS channel probing, only one of the backlogged users with channel quality better than certain level is allowed to access media. By reusing collision avoidance handshake, the overhead to utilize diversity is very small [5].

The latest technique to improve the disseminating performance is to allow each node solicits and cache the unsubscribed contents according to its available buffer so that it can better serve its potential encounters in the future. Therefore, by considering two encountering nodes as a group, a novel cooperative content dissemination framework (CCDF) was proposed which offers group based optimal disseminating decisions to maximize the overall content served performance even under restricted resource conditions such as storage space and link bandwidth [11].

\section{OBSERVATIONS}

The energy efficient opportunistic topology control can significantly improve energy efficiency. Also it minimizes the number of coordinator and satisfies given requirement on end-to-end network performance [3]. Also we observe that transitional links can make an important impact on the design of QoS topology control algorithms. We introduce opportunistic communication in topology control for WSNs by considering transitional links. Catching the transmission opportunities provided by the transitional links, opportunistic transmission can significantly improve energy efficiency in QoS topology control with low communication overhead [1]. The another potential issue is how to integrate opportunistic forwarding with the emerging multi-channel multi-radio (MCMR) networks, where additional spectrum diversity has to be coordinated with the spatial and temporal diversity provided by opportunistic forwarding [7].

Weakness of many existing schemes is their reliance on end-to-end routing knowledge overlay. While independent algorithms entirely forsaking routing yet being adaptive to end-to-end topology changes are highly desirable in terms of network resilience and forwarding efficiency.

There is routing protocol for Disruption Tolerant Networks (DTNs) because of this there is some reduction in routing 
overhead which help in improving the routing performance [4]. With the help of cooperative and opportunistic transmission as well as by using credit calculation and flow scheduling we can achieve the cooperation of the entire transmitters which leads to improve the overall performance and throughput [8].

Challenge is the appropriate design balance and trade off in terms of cross-layer (MAC and network) forwarding co-operation. More MAC-intrusive design may lead to smaller forwarding latency but it also limits the applicability of the technology. The error control can be achieved in wireless ad hoc network with multiple co-operative relays [6]. As well as the ROSA framework improves retransmission technique by moving retransmission decision from MAC protocol to scheduler, also it improves the communication by the node cooperation and considering the link status [9]. A cooperative content dissemination framework (CCDF) is used to share contents among mobile users by utilizing low cost wireless connectivity as well as storage spaces efficiently [11] Multiuser diversity and high quality channel duration can be significantly exploited in CSMA/CA based WLANs and multihop ad hoc networks by opportunistic media access and rate adaptation protocol (OSAR) [5]. By exploiting the generalized multiuser diversity, i.e. by assigning the links in better channel conditions with higher priority to access the channel, the network throughput can be dynamically increased [10]

\section{PROPOSED IDEA}

To transfer the data from source to destination in wireless and mobile ad hoc network opportunistic transmission is useful, in which the packets are being send to neighbouring nodes. These neighbouring nodes again send those packets to their neighbouring nodes and so on. In this method if opportunistically the receiver found then packet will be forwarded to receiver. So in opportunistic network the packet flooding is major issue. To overcome this issue there are various routing and forwarding protocols had been derived.

\section{A. Mathematical Solution}

Let us design wireless environment first,

System:

$$
\begin{array}{ll}
S=\{D, N, P & \\
D=\left\{D_{1}, D_{2}, \ldots \ldots, D_{n}\right\} & \text { is set of Data } \\
N=\left\{N_{1}, N_{2}, \ldots \ldots, N_{k}\right\} & \text { is set of All Neighbouring } \\
P=\left\{P_{1}, P_{2}, \ldots \ldots, P_{m}\right\} & \begin{array}{l}
\text { Nodes } \\
\text { is set of neighbouring nodes } \\
\text { which can transfer packet } \\
\text { towards destination }
\end{array}
\end{array}
$$

where $\mathrm{m}<=\mathrm{k}$

$$
\begin{aligned}
& \text { Input: } \\
& \begin{array}{l}
\mathrm{D} \text { or } \mathrm{Di} \\
\mathrm{N}=\left\{\mathrm{N}_{1}, \mathrm{~N}_{2}, \ldots \ldots . \mathrm{N}_{\mathrm{k}}\right\} \\
\mathrm{P}=\left\{\mathrm{P}_{1}, \mathrm{P}_{2}, \ldots \ldots, \mathrm{P}_{\mathrm{m}}\right\}
\end{array}
\end{aligned}
$$

Output:

$$
\text { Source }_{\text {sent }}(\mathrm{D})=\text { Destination }_{\text {received }}(\mathrm{D})
$$

\author{
Procedure: \\ Source node: \\ $\operatorname{Send}\left(\mathrm{D}_{\mathrm{i}}, \mathrm{P}_{\mathrm{j}}\right)$ \\ Intermediate nodes: \\ $\operatorname{Receive}\left(\mathrm{D}_{\mathrm{i}}\right)$ \\ $\operatorname{Send}\left(\mathrm{D}_{\mathrm{i}}, \mathrm{P}_{\mathrm{j}}\right)$ \\ Destination node : \\ $\operatorname{Receive}\left(\mathrm{D}_{\mathrm{i}}\right)$
}

\section{B. Mathematical Solution}

Here new idea is every node will keep the record of all neighbouring nodes, where record will give information about which neighbouring node will help to reach which destination. In this transmission every node in route will participate in decision making (on the basis of record) to transmit data, which will automatically find route up to destination. This route is based on the connected end-to-end path in previous transmission when record of neighbouring node is created or updated. In this set of route if any link is disconnected then possibility to reconnect the link is more than establishing any new link. If, the receiver get disconnected on any link there will be high possibility to reconnect on same link then for sending packet to receiver we can give higher priority to all end-to-end known path and by using these paths with opportunistic transmission we can improve throughput, because the probability of reconnection of receiver on disconnected links is more.

\section{CONCLUSION}

This paper gives overview of the cooperative and opportunistic transmission techniques and implies some drawback and advantages of the existing techniques.

We had proposed one IDEA based on routing mechanism for further research.

\section{REFERENCES}

[1] J. Ma, C. Qian, Q. Zhang, and L. Ni, "Opportunistic transmission based QoS topology control in wireless sensor networks," in Proc. 5th IEEE International Conference on Mobile Ad Hoc and Sensor Systems, 2008. MASS 2008, 2008, pp. 422-427. doi:10.1109/MAHSS.2008.4660050.

[2] L. Pelusi, A. Passarella, and M. Conti, "Opportunistic networking: data forwarding in disconnected mobile ad hoc networks," IEEE Communications Magazine, vol. 44, no. 11, November 2006.

[3] J. Ma, Q. Zhang, C. Qian, and L. M. Ni, "Energy-efficient opportunistic topology control in wireless sensor networks," in Proc. ACM / SIGMOBILE MobiOpp 2007 in conjunction with MobiSys, 2007

[4] M. Caleffi and L. Paura, "Opportunistic routing for disruption tolerant networks," in Proc. AINA 09: the IEEE 23rd International Conference on Advanced Information Networking and Applications, May 2009.

[5] J. Wang, H. Zhai, Y. Fang, and M. C. Yuang, "Opportunistic media access control and rate adaptation for wireless ad hoc networks," in Proc. IEEE ICC'04, June, 2004.

[6] H. Rutagemwa and T. Willink, "Throughput and spectral efficiency in ARQ based cooperative ad hoc networks," IEEE, 2010.

[7] H. Zhu and K. Lu, "Resilient opportunistic forwarding: Issues and challenges," in Proc. Military Communications Conference, 2007. IEEE, pp. 1-7, Oct. 2007.

[8] Q. Zhang, Q. Chen, F. Yang, X. Shen, and Z. Niu, "Cooperative and opportunistic transmission for wireless ad hoc networks," IEEE Network, vol. 21, no. 1, pp. 14-20, 2007. 
[9] Y. Sun, V. O. K. Li, and K.-C. Leung, "Distributed opportunistic scheduling in multihop wireless Ad Hoc networks," IEEE, 2008.

[10] Q. Chen, F. Ye, and Z. Niu, "Graph theoretical analysis of opportunistic scheduling policy for wireless ad hoc networks," in Proc. IEEE Globecom, November 2007.

[11] Y. Ma and A. Jamalipour, "Cooperative content dissemination in intermittently connected networks," in Proc. IEEE ICC'09, Dresden, Germany, Jun 2009.

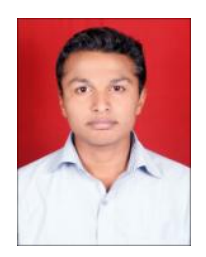

Suyog Suresh Nyati was born in pachora (Jalgaon, Maharashtra, India) on 11th Oct., 1988. He is pursuing Master's Degree in 'Computer Engineering' from 'Pune Institute of Computer Technology, Pune'. He has completed bachelors in Computer Science \& Engineering (BE CSE) in 2010 from 'G. H. Raisoni college, Pune, India.

His research interests lie in Computer Networking. He has recently published research paper-"Co-operative and Opportunistic Transmission in Mobile and Ad-Hoc Networks" The paper proposes a new idea of routing packets in mobile and ad-hoc networks.

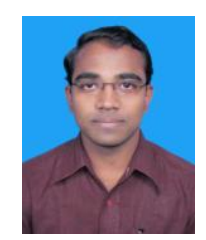

Suresh Limkar received B.E. (Computer Science \&Engineering) and M.E. (Computer Engineering) degree in 2005 and 2007 respectively. He is presently working as an assistant professor in Computer Engineering Department of AISSMS's IOIT, Pune. He has published his research papers in several International Conferences and International Journal. His research interest includes Network Security, Location based services, Security in Location based service 